\title{
RISKS OF TRANSPORTATION ALONG VARIOUS ROUTES TO THE NEVADA TEST SITE
}

Ruth F. Weiner

Sandia National Laboratories

\section{INTRODUCTION}

Residents of Southern Nevada have expressed interest in the risks posed by transportation of radioactive materials to the Nevada Test Site for disposal. Residents of the urban sections of Clark County, in particular, are concerned because the trucks carrying this material pass through fairly densely populated areas on both primary and secondary highways. Excellent studies by the Desert Research Institute (Miller, et al, 2005; 2007) measured the external radiation doses from these trucks and provided a benchmark for estimating the doses sustained by the population along the transportation routes. The studies did not, however, assess the doses to the population in the event of anaccident involving these trucks. The present study estimates doses to the population along these routes and to various individual receptors, both for routine, incident-free transportation and for transportation accidents.

\section{STUDY METHODS}

\section{RADTRAN}

The program and code RADTRAN was used to estimate transportation risks (Neuhauser, et al, 2000; Weiner et al, 2006). RADTRAN estimates doses from routine, incident-free transportation by modeling the radioactive cargo as a sphere moving at the speed of the vehicle; the external dose rate at one meter from the cargo (the transport index, or $\mathrm{TI}$ ) is modeled as a virtual radiation source at the center of this sphere. Doses from transportation accidents that do not involve releases of ionizing radiation also use this model. Accidents in which radioactive material is assumed to be released are modeled by Gaussian dispersion and deposition of released material. Details of these models may be found in Neuhauser et al (2000) and Weiner et al (2006). RADTRAN 5.6 with the input file generator RADCAT 2.3 was used for this study.

RADTRAN input data are shown in Tables 1, 2 and 3. All transportation was by truck. Table 1 Shows the parameters related to population, crew, accidents, vehicle speeds, etc. 
Table 1. Parameters used in calculating incident-free doses.

\begin{tabular}{|c|c|c|}
\hline Parameter & Parameter Value & Comments and Reference \\
\hline \multicolumn{3}{|l|}{ Package } \\
\hline Package type & Type A & \\
\hline Package dimension & See Table 2 & INEL04SMC002. 0001; NFSI1000000002, 3 \\
\hline External Dose rate (TI) & See Table 2 & INEL04SMC002. 0001; NFSI1000000002, 3 \\
\hline $\begin{array}{l}\text { Fraction of emitted radiation that is } \\
\text { gamma }\end{array}$ & $1.00(0.99998)$ & \multirow[t]{2}{*}{$\begin{array}{l}\text { Calculated from inventory and ICRP38 } \\
\text { (1983) }\end{array}$} \\
\hline $\begin{array}{l}\text { Fraction of emitted radiation that is } \\
\text { neutrons }\end{array}$ & $2 \times 10^{-5}$ & \\
\hline \multicolumn{3}{|l|}{ Crew } \\
\hline Number of crew & 2 & \\
\hline Distance from source to crew & $3.1 \mathrm{~m}$ & \\
\hline Dose to truck crew during travel & $2 \mathrm{mrem} / \mathrm{hr}$ & This is the regulatory maximum. \\
\hline \multicolumn{2}{|l|}{ Truck Speeds } & \multirow{4}{*}{$\begin{array}{l}\text { Insurance Institute for Highway Safety, as } \\
\text { cited by the U.S. Bureau of Transportation } \\
\text { Statistics, State Transportation Statistics } \\
\text { 2005, Table 2-9 (www.bts.gov) }\end{array}$} \\
\hline Rural & $113 \mathrm{~km}^{\prime} \mathrm{hr}^{\mathrm{a}}$ & \\
\hline Suburban & $113 \mathrm{~km} / \mathrm{hr}$ & \\
\hline Urban & $\begin{array}{l}105 \mathrm{~km} / \mathrm{hr} ; \\
\text { Clark Co: } 97 \mathrm{~km} / \mathrm{hr}\end{array}$ & \\
\hline Traffic accident rate & $3.6 \times 10^{-6} /$ vehicle-km & \multirow{2}{*}{$\begin{array}{l}\text { Nevada data, State Transportation } \\
\text { Statistics, U.S. Bureau of Transportation } \\
\text { Statistics, 2005, Table 2-1 (www.bts.gov) }\end{array}$} \\
\hline Traffic fatality rate & $1.27 \times 10^{-8} /$ vehicle-km & \\
\hline $\begin{array}{l}\text { Number of people per vehicle } \\
\text { sharing route }\end{array}$ & 2 & \\
\hline $\begin{array}{l}\text { Minimum and maximum distances } \\
\text { to exposed resident population along } \\
\text { the route }\end{array}$ & 30 to $800 \mathrm{~m}$ & \\
\hline \multicolumn{3}{|l|}{ Population densities (persons per $\mathrm{km}^{2}$ ) } \\
\hline Rural & See Table 3 & \\
\hline Suburban & See Table 3 & \\
\hline Urban & See Table 3 & \\
\hline \multicolumn{3}{|c|}{ One-way traffic count (vehicles per hour) on Nevada highways } \\
\hline Clark Co. Rural & 3640 & \multirow{6}{*}{$\begin{array}{l}\text { From The Transportation Health and Safety } \\
\text { Calculation Package, DOE, 2002. The traffic } \\
\text { counts for counties other than Clark Co are } \\
\text { the average for the state (excluding Clark } \\
\text { Co.) because there were not enough data } \\
\text { for each of the other counties. }\end{array}$} \\
\hline Clark Co. Suburban & 1230 & \\
\hline Clark Co. Urban & 3160 & \\
\hline Other Nevada Rural & 148 & \\
\hline Other Nevada Suburban & 311 & \\
\hline Other Nevada Urban & 2010 & \\
\hline
\end{tabular}

${ }^{\mathrm{a}} 1 \mathrm{~km}=0.6217 \mathrm{mi}$.

Table 2 shows the parameters related to the cargo section of the vehicle. 
Table 2. Cargo parameters used in calculating incident-free doses.

\begin{tabular}{|l|l|c|c|c|}
\hline & & \multicolumn{2}{|c|}{ Vehicle (Cargo) Dimensions (m) } & \\
\hline Shipment ID & Material & Length & Crew View $^{\text {a }}$ & TI(mrem/hr \\
\hline INL08001 & unknown & 8.41 & 3.66 & 0.001 \\
\hline INL08002 & unknown & 6.06 & 3.14 & 0.50 \\
\hline INL08003 & unknown & 6.06 & 3.14 & 0.01 \\
\hline NFL08041 & caustic & 8.41 & 3.66 & 0.01 \\
\hline NFL08042 & caustic & 8.41 & 3.66 & 1.00 \\
\hline NFL08043 & raffinate & 8.41 & 3.66 & 0.01 \\
\hline POL08131 & scrap metal & 8.41 & 3.66 & 0.01 \\
\hline POL08132 & scrap metal & 8.41 & 3.66 & 0.10 \\
\hline POL08133 & scrap metal & 8.41 & 3.66 & 0.01 \\
\hline POL08134 & scrap metal & 8.41 & 3.66 & 0.10 \\
\hline PORTLP0001002 & $\begin{array}{l}\text { wet solid ,HEPA } \\
\text { filters }\end{array}$ & 6.10 & 3.14 & 2.80 \\
\hline NFS100000000302 & $\begin{array}{l}\text { stabilized } \\
\text { caustic }\end{array}$ & 6.10 & 3.56 & 10.00 \\
\hline NFS100000000201 & $\begin{array}{l}\text { stabilized } \\
\text { raffinate }\end{array}$ & 6.10 & 3.56 & \\
\hline
\end{tabular}


Table 3. Radionuclide inventories in curies for each package shipped.

\begin{tabular}{|c|c|c|c|c|c|c|c|c|c|c|c|c|c|c|c|}
\hline Shipment ID & NP237 & PA234M & PU238 & PU239 & TC99 & TH231 & TH234 & U232 & U234 & U235 & U236 & U238 & AM241 & PA231 & RA226 \\
\hline INL08001 & $5.65 \mathrm{E}-10$ & 8.57E-05 & $8.57 \mathrm{E}-11$ & $1.28 \mathrm{E}-10$ & $4.89 \mathrm{E}-08$ & $1.08 \mathrm{E}-06$ & 8.57E-05 & $1.50 \mathrm{E}-07$ & $1.54 \mathrm{E}-05$ & $1.08 \mathrm{E}-06$ & $5.00 \mathrm{E}-07$ & 8.57E-05 & $8.86 \mathrm{E}-10$ & & \\
\hline INL08002 & $1.20 \mathrm{E}-06$ & 1.07E-01 & $6.60 \mathrm{E}-06$ & 8.54E-07 & $1.75 \mathrm{E}-04$ & 1.07E-01 & 1.07E-01 & $1.84 \mathrm{E}-04$ & $5.26 \mathrm{E}-02$ & $1.30 \mathrm{E}-03$ & $1.34 \mathrm{E}-05$ & 1.07E-01 & $2.70 \mathrm{E}-11$ & & \\
\hline INL08002 & $1.76 \mathrm{E}-06$ & $1.57 \mathrm{E}-01$ & $9.69 \mathrm{E}-06$ & $1.25 \mathrm{E}-06$ & $2.56 \mathrm{E}-04$ & $1.92 \mathrm{E}-03$ & 1.57E-01 & $2.70 \mathrm{E}-04$ & 7.74E-02 & $1.92 \mathrm{E}-03$ & $1.97 \mathrm{E}-03$ & 1.57E-01 & $9.14 \mathrm{E}-06$ & & \\
\hline INL08002 & $1.20 \mathrm{E}-06$ & 1.07E-01 & $6.60 \mathrm{E}-06$ & $8.54 \mathrm{E}-07$ & $1.75 \mathrm{E}-04$ & $1.30 \mathrm{E}-03$ & 1.07E-01 & $1.84 \mathrm{E}-04$ & $5.26 \mathrm{E}-02$ & $1.30 \mathrm{E}-03$ & $1.34 \mathrm{E}-03$ & 1.07E-01 & $6.23 \mathrm{E}-06$ & & \\
\hline INL08002 & 1.76E-06 & $1.57 \mathrm{E}-01$ & 9.69E-06 & $1.25 \mathrm{E}-06$ & $2.56 \mathrm{E}-04$ & $1.92 \mathrm{E}-03$ & 1.57E-01 & $2.70 \mathrm{E}-04$ & 7.74E-02 & $1.92 \mathrm{E}-03$ & 1.97E-03 & 1.57E-01 & $9.14 \mathrm{E}-06$ & & \\
\hline INL08002 & $1.20 \mathrm{E}-06$ & 1.07E-01 & $6.60 \mathrm{E}-06$ & 8.54E-07 & $1.75 E-04$ & 1.30E-03 & 1.07E-01 & $1.84 \mathrm{E}-04$ & $5.26 \mathrm{E}-02$ & $1.30 \mathrm{E}-03$ & $1.34 \mathrm{E}-03$ & 1.07E-01 & $6.23 \mathrm{E}-06$ & & \\
\hline INL08002 & $1.76 \mathrm{E}-06$ & 1.57E-01 & 9.69E-06 & $1.25 \mathrm{E}-06$ & $2.56 \mathrm{E}-04$ & $1.92 \mathrm{E}-03$ & 1.57E-01 & $2.70 \mathrm{E}-04$ & 7.74E-02 & $1.92 \mathrm{E}-03$ & $1.97 \mathrm{E}-03$ & 1.57E-01 & $9.14 \mathrm{E}-06$ & & \\
\hline INL08002 & $1.20 \mathrm{E}-06$ & 1.07E-01 & $6.60 \mathrm{E}-06$ & 8.54E-07 & $1.75 \mathrm{E}-04$ & 1.30E-03 & 1.07E-01 & $1.84 \mathrm{E}-04$ & $5.26 \mathrm{E}-02$ & 1.30E-03 & $1.34 \mathrm{E}-03$ & 1.07E-01 & $6.23 \mathrm{E}-06$ & & \\
\hline INL08002 & 1.76E-06 & $1.57 \mathrm{E}-01$ & 9.69E-06 & 1.25E-06 & $2.56 \mathrm{E}-04$ & $1.92 \mathrm{E}-03$ & 1.57E-01 & 2.70E-04 & 7.74E-02 & $1.92 \mathrm{E}-03$ & $1.97 \mathrm{E}-03$ & 1.57E-01 & $9.14 \mathrm{E}-06$ & & \\
\hline INL08002 & $1.20 \mathrm{E}-06$ & 1.07E-01 & $6.60 \mathrm{E}-06$ & 8.54E-07 & $1.75 \mathrm{E}-04$ & 1.30E-03 & 1.07E-01 & $1.84 \mathrm{E}-04$ & $5.26 \mathrm{E}-02$ & 1.30E-03 & $1.34 \mathrm{E}-03$ & 1.07E-01 & $6.23 \mathrm{E}-06$ & & \\
\hline INL08002 & $1.76 \mathrm{E}-06$ & $1.57 \mathrm{E}-01$ & 9.69E-06 & $1.25 \mathrm{E}-06$ & $2.56 \mathrm{E}-04$ & $1.92 \mathrm{E}-03$ & 1.57E-01 & $2.70 \mathrm{E}-04$ & 7.74E-02 & $1.92 \mathrm{E}-03$ & $1.97 \mathrm{E}-03$ & 1.57E-01 & $9.14 \mathrm{E}-06$ & & \\
\hline INL08002 & $1.20 \mathrm{E}-06$ & $1.07 \mathrm{E}-01$ & $6.60 \mathrm{E}-06$ & $8.54 \mathrm{E}-07$ & $1.75 \mathrm{E}-04$ & $1.30 \mathrm{E}-03$ & 1.07E-01 & $1.84 \mathrm{E}-04$ & $5.26 \mathrm{E}-02$ & $1.30 \mathrm{E}-03$ & $1.34 \mathrm{E}-03$ & 1.07E-01 & $6.23 \mathrm{E}-06$ & & \\
\hline INL08002 & 1.76E-06 & $1.57 \mathrm{E}-01$ & $9.69 \mathrm{E}-06$ & $1.25 \mathrm{E}-06$ & $2.56 \mathrm{E}-04$ & $1.92 \mathrm{E}-03$ & 1.57E-01 & $2.70 \mathrm{E}-04$ & 7.74E-02 & $1.92 \mathrm{E}-03$ & 1.97E-03 & 1.57E-01 & $9.14 \mathrm{E}-06$ & & \\
\hline INL08002 & $1.20 \mathrm{E}-06$ & 1.07E-01 & $6.60 \mathrm{E}-06$ & $8.54 \mathrm{E}-07$ & $1.75 \mathrm{E}-04$ & 1.30E-03 & 1.07E-01 & $1.84 \mathrm{E}-04$ & $5.26 \mathrm{E}-02$ & 1.30E-03 & $1.34 \mathrm{E}-03$ & 1.07E-01 & $6.23 \mathrm{E}-06$ & & \\
\hline INL08002 & $1.76 \mathrm{E}-06$ & 1.57E-01 & $9.69 \mathrm{E}-06$ & $1.25 \mathrm{E}-06$ & $2.56 \mathrm{E}-04$ & $1.92 \mathrm{E}-03$ & 1.57E-01 & $2.70 \mathrm{E}-04$ & 7.74E-02 & $1.92 \mathrm{E}-03$ & 1.97E-03 & 1.57E-01 & $9.14 \mathrm{E}-06$ & & \\
\hline INL08002 & $1.20 \mathrm{E}-06$ & 1.07E-01 & $6.60 \mathrm{E}-06$ & 8.54E-07 & $1.75 \mathrm{E}-04$ & 1.30E-03 & 1.07E-01 & $1.84 \mathrm{E}-04$ & $5.26 \mathrm{E}-02$ & 1.30E-03 & $1.34 \mathrm{E}-03$ & 1.07E-01 & $6.23 \mathrm{E}-06$ & & \\
\hline INL08002 & $1.76 \mathrm{E}-06$ & 1.57E-01 & $9.69 \mathrm{E}-06$ & $1.25 \mathrm{E}-06$ & $2.56 \mathrm{E}-04$ & 1.92E-03 & 1.57E-01 & $2.70 \mathrm{E}-04$ & 7.74E-02 & $1.92 \mathrm{E}-03$ & $1.97 \mathrm{E}-03$ & 1.57E-01 & $9.14 \mathrm{E}-06$ & & \\
\hline INL08002 & $1.20 \mathrm{E}-06$ & 1.07E-01 & $6.60 \mathrm{E}-06$ & $8.54 \mathrm{E}-07$ & $1.75 \mathrm{E}-04$ & $1.30 \mathrm{E}-03$ & 1.07E-01 & $1.84 \mathrm{E}-04$ & $5.26 \mathrm{E}-02$ & $1.30 \mathrm{E}-03$ & $1.34 \mathrm{E}-03$ & 1.07E-01 & $6.23 \mathrm{E}-06$ & & \\
\hline INL08002 & $1.76 \mathrm{E}-06$ & 1.57E-01 & $9.69 \mathrm{E}-06$ & $1.25 \mathrm{E}-06$ & $2.56 \mathrm{E}-04$ & $1.92 \mathrm{E}-03$ & 1.57E-01 & $2.70 \mathrm{E}-04$ & 7.74E-02 & $1.92 \mathrm{E}-03$ & $1.97 \mathrm{E}-03$ & $1.57 \mathrm{E}-01$ & $9.14 \mathrm{E}-06$ & & \\
\hline INL08002 & $1.20 \mathrm{E}-06$ & 1.07E-01 & $6.60 \mathrm{E}-06$ & $8.54 \mathrm{E}-07$ & $1.75 \mathrm{E}-04$ & 1.30E-03 & 1.07E-01 & $1.84 \mathrm{E}-04$ & $5.26 \mathrm{E}-02$ & 1.30E-03 & $1.34 \mathrm{E}-03$ & 1.07E-01 & $6.23 \mathrm{E}-06$ & & \\
\hline INL08002 & $1.76 \mathrm{E}-06$ & $1.57 \mathrm{E}-01$ & $9.69 \mathrm{E}-06$ & $1.25 \mathrm{E}-06$ & $2.56 \mathrm{E}-04$ & $1.92 \mathrm{E}-03$ & 1.57E-01 & $2.70 \mathrm{E}-04$ & $7.74 \mathrm{E}-02$ & $1.92 \mathrm{E}-03$ & $1.97 \mathrm{E}-03$ & 1.57E-01 & $9.14 \mathrm{E}-06$ & & \\
\hline INL08002 & $1.20 \mathrm{E}-06$ & $1.07 \mathrm{E}-01$ & $6.60 \mathrm{E}-06$ & $8.54 \mathrm{E}-07$ & $1.75 \mathrm{E}-04$ & $1.30 \mathrm{E}-03$ & 1.07E-01 & $1.84 \mathrm{E}-04$ & $5.26 \mathrm{E}-02$ & $1.30 \mathrm{E}-03$ & $1.34 \mathrm{E}-03$ & 1.07E-01 & $6.23 \mathrm{E}-06$ & & \\
\hline INL08002 & $1.76 \mathrm{E}-06$ & $1.57 \mathrm{E}-01$ & $9.69 \mathrm{E}-06$ & $1.25 \mathrm{E}-06$ & $2.56 \mathrm{E}-04$ & $1.92 \mathrm{E}-03$ & 1.57E-01 & $2.70 \mathrm{E}-04$ & 7.74E-02 & $1.92 \mathrm{E}-03$ & $1.97 \mathrm{E}-03$ & 1.57E-01 & $9.14 \mathrm{E}-06$ & & \\
\hline INL08002 & $1.20 \mathrm{E}-06$ & 1.07E-01 & $6.60 \mathrm{E}-06$ & 8.54E-07 & $1.75 E-04$ & 1.30E-03 & 1.07E-01 & $1.84 \mathrm{E}-04$ & $5.26 \mathrm{E}-02$ & $1.30 \mathrm{E}-03$ & $1.34 \mathrm{E}-03$ & 1.07E-01 & $6.23 \mathrm{E}-06$ & & \\
\hline INL08002 & $1.76 \mathrm{E}-06$ & $1.57 \mathrm{E}-01$ & $9.69 \mathrm{E}-06$ & $1.25 \mathrm{E}-06$ & $2.56 \mathrm{E}-04$ & $1.92 \mathrm{E}-03$ & 1.57E-01 & $2.70 \mathrm{E}-04$ & 7.74E-02 & $1.92 \mathrm{E}-03$ & $1.97 \mathrm{E}-03$ & 1.57E-01 & $9.14 \mathrm{E}-06$ & & \\
\hline NFL08041 & & & & & & & & & $3.62 \mathrm{E}-02$ & 7.41E-04 & $8.81 \mathrm{E}-03$ & $2.22 \mathrm{E}-05$ & & & \\
\hline NFL08042 & & & & & & & & & $2.81 \mathrm{E}-02$ & $5.73 \mathrm{E}-04$ & $6.81 \mathrm{E}-03$ & $1.72 \mathrm{E}-05$ & & & \\
\hline NFL08043 & $6.57 \mathrm{E}-04$ & & $4.86 \mathrm{E}-06$ & & $3.11 \mathrm{E}-03$ & & & & $1.15 \mathrm{E}-02$ & & & $2.62 \mathrm{E}-03$ & & $2.38 \mathrm{E}-03$ & 2.86E-03 \\
\hline POL08131 & & & & & 1.49E-03 & & & & $1.93 \mathrm{E}-04$ & $6.67 \mathrm{E}-06$ & & 4.04E-05 & & & \\
\hline POL08132 & & & & & $1.15 E-03$ & & & & $2.48 \mathrm{E}-04$ & $8.58 \mathrm{E}-06$ & & 5.19E-05 & & & \\
\hline POL08133 & & & & & $1.67 \mathrm{E}-03$ & & & & 5.57E-04 & 1.93E-05 & & 1.17E-04 & & & \\
\hline POL08134 & & & & & $1.88 \mathrm{E}-03$ & & & & 3.13E-04 & $1.08 \mathrm{E}-05$ & & 6.64E-05 & & & \\
\hline PORTLP0001002 & & & & & 2.19E-04 & & & & $2.56 \mathrm{E}-04$ & $1.48 \mathrm{E}-05$ & & $2.28 \mathrm{E}-04$ & & & \\
\hline NFS100000000302 & & & & & & & & & 7.97E-02 & $1.64 \mathrm{E}-03$ & $1.94 \mathrm{E}-02$ & 5.50E-05 & & & \\
\hline NFS100000000201 & $6.80 \mathrm{E}-06$ & & $7.20 \mathrm{E}-06$ & & & & & & $5.40 \mathrm{E}-03$ & & $1.25 \mathrm{E}-03$ & & $9.80 \mathrm{E}-06$ & $1.13 \mathrm{E}-03$ & $1.33 \mathrm{E}-03$ \\
\hline
\end{tabular}





\section{REFERENCES}

International Commission on Radiation Protection (ICRP) 38. 1983. Radionuclide Transformations: Energy and Intensity of Emissions. Pergamon Press, New York, NY.

Miller, J.J., Shafer, D.S. Gray, K. J. Church, B. W., Campbell, S. A., Holz, B.A. 2005. Assessing Potential Exposure from Truck Transport of LowLevel Radioactive Waste to the Nevada Test Site, DOE/NV 13609-37, Desert Research Institute, Las Vegas, NV.

Miller, J.J., Shafer, D.S. Gray, K. J. Church, B. W., Campbell, S. A., Holz-Montemayor, B. 2007. "Characterizing Potential Exposure to the Public from Low-Level Radioactive Waste Transportation by Truck," Health Physics v. 93 pp 6 et seq.

Neuhauser, K.S.; Kanipe, F.L.; and Weiner, R.F. 2000. RADTRAN 5 Technical Manual. SAND2000-1256. Sandia National Laboratories, Albuquerque, NM.

U.S. Department of Energy (DOE). 2002. Final Environmental Impact Statement for a Geologic Repository for the Disposal of Spent Nuclear Fuel and High-Level Radioactive Waste at Yucca Mountain, Nye County, Nevada. DOE/EIS-0250-F. Summary, Volumes I and II. Washington, D.C.: U.S. Department of Energy, Office of Civilian Radioactive Waste Management.

Weiner, R.F., Osborn, D.M., Mills, G.S., Hinojosa, D, Heames, T.J., Orcutt, D.J. 2006. RadCat 2.3 User Guide SAND2006-6315. Sandia National Laboratories, Albuquerque, NM. 